\title{
Anti-Staphylococcal humoral immune response in patients with chronic rhinosinusitis*
}

\section{Ulrica Thunberg ${ }^{1,2}$, Svante Hugosson 1,2, Hans Fredlund ${ }^{2,3}$, Yang Cao ${ }^{4,5}$, Ralf Ehricht ${ }^{6,7,8}$, Stefan Monecke ${ }^{6,7,9}$, Elke Müller ${ }^{6,7}$, Susanne Engelmann ${ }^{10,11}$, Bo Söderquist ${ }^{2,3}$}

Rhinology Online, Vol 2: 50 - 58, 2019

http://doi.org/10.4193/RHINOL/19.002

*Received for publication:

January 13, 2019

Accepted: March 5, 2019

Published: March 22, 2019

\footnotetext{
2 Faculty of Medicine and Health, Örebro University, Sweden

${ }^{3}$ Department of Laboratory Medicine, Clinical Microbiology, Örebro University Hospital, Örebro, Sweden

${ }^{4}$ Clinical Epidemiology and Biostatistics, School of Medical Sciences, Örebro University, Örebro, Sweden

Unit of Biostatistics, Institute of Environmental Medicine, Karolinska Institute, Stockholm, Sweden

${ }^{6}$ Abbott (Alere Technologies GmbH), Jena, Germany

InfectoGnostics Research Campus, Jena, Germany

${ }^{8}$ Leibniz Institute of Photonic Technology (IPHT), Jena, Germany

9 Institute for Medical Microbiology and Hygiene, Technical University of Dresden, Dresden, Germany

${ }^{10}$ Institute for Microbiology, Technical University Braunschweig, Germany

${ }^{1}$ Microbial Proteomics, Helmholtz Center for Infection Research, Braunschweig, Germany
}

\begin{abstract}
Background: Staphylococcus aureus (S. aureus) can behave both as a harmless commensal and as a pathogen. Its significance in the pathogenesis of chronic rhinosinusitis (CRS) is not yet fully understood. This study aimed to determine serum antibody responses to specific staphylococcal antigens in patients with CRS and healthy controls, and to investigate the correlation between specific antibody response and severity of symptoms.
\end{abstract}

Methodology: Serum samples from 39 patients with CRS and 56 healthy controls were analysed using a protein microarray to investigate the antibody response to $S$. aureus specific antigens, with a focus on immunoglobulin $\mathrm{G}$ (IgG) directed toward staphylococcal components accessible to the immune system. Holm-Bonferroni corrections were applied in all analyses. Information about growth of $S$. aureus in nares and maxillary sinus was taken from a previous study based on the same individuals. Clinical symptoms were assessed using a scoring system.

Results: IgG antibody levels toward staphylococcal TSST-1 and LukF-PV were significantly higher in the CRS patient group compared to healthy controls, and levels of anti-TSST-1 antibodies were significantly higher in the CRS patient group with S. aureus in maxillary sinus than in controls. There were no correlations between the severity of symptoms and levels of serum anti-staphylococcal lgG antibody levels for LukF-PV and TSST-1.

Conclusions: TSST-1 and LukF-PV could be interesting markers for future studies of the pathogenesis of CRS.

Key words: antibodies, chronic rhinosinusitis, immunoglobulin, G, protein microarray, staphylococcal antigen

\section{Introduction}

Chronic rhinosinusitis (CRS) is a common disease that affects about $10 \%$ of the European population, according to a large questionnaire study by the Global Allergy and Asthma European
Network (GA2LEN) (1). Its cause is not known, but studies have suggested the involvement of Staphylococcus aureus ${ }^{(2-4)}$. In normal maxillary sinuses, $S$. aureus is rare and is usually regarded as an insignificant finding, but the bacteria have been reported 
in more than $25 \%$ of patients with CRS ${ }^{(5,6)}$. About $20 \%$ (range: $12-30 \%$ ) of the population persistently carries $S$. aureus in the nares, and approximately 30\% (range: 16-70\%) of the rest are intermittent carriers ${ }^{(7,8)}$. Beyond being a harmless commensal, $S$. aureus is able to cause various diseases ranging from mild to severe and even fatal conditions. There is an impact of $S$. aureus in other chronic inflammatory diseases, such as atopic dermatitis $(9$, ${ }^{10)}$ and asthma ${ }^{(11)}$. It has been proposed that in ulcerative colitis a preexisting CRS may affect the colitis by swallowing of sinusitis derived S. aureus antigens ${ }^{(12)}$. The bacteria display a wide arsenal of virulence factors that can contribute to the pathogenesis of an infection ${ }^{(13)}$. Superantigens such as toxic shock syndrome toxin-1 (TSST-1) and enterotoxins have been suggested to play a role in CRS, especially in CRS with nasal polyposis (CRSwNP) ${ }^{(3,13)}$.

In this study, we focused on $\lg G$ antibodies directed against specific components of $S$. aureus, such as surface adhesins and secreted toxins, which are of benefit in host defence against staphylococcal infections. Humoral immune deficiency has been shown to be a common condition in patients with medically refractory CRS ${ }^{(14-16)}$. The individual antibody profile might be of importance for the clinical course/severity of CRS. A review by Van Belkum et al. concluded that there is a need for further studies of host factors that might be able to mitigate the effects of staphylococcal toxins in disease ${ }^{(17)}$. The aim of the present study was to use protein microarray technology to evaluate antibody responses to specific staphylococcal antigens, especially towards enterotoxins, in serum from patients with CRS and healthy controls. Another aim was to investigate the correlation between severity of symptoms and the specific antibody response. Given knowledge of anti-staphylococcal lgG antibody responses to $S$. aureus antigens in CRS patients and healthy controls, it might be possible to identify specific biomarkers that could be useful in future studies of the course of the disease and its treatment.

\section{Materials and methods}

\section{Patients and controls}

Forty-two patients with CRS and 57 healthy controls were included between 2004 and 2010. Two physicians specializing in otorhinolaryngology (UT and SH) performed all inclusion procedures. All patients had been diagnosed with CRS for more than 1 year (range 1-9 years: median: 3 years) prior to inclusion. The CRS diagnosis was based on history, clinical examination, and CT scans according to the definitions and guidelines of the position paper on rhinosinusitis ${ }^{(18)}$ prepared by the Academy of Allergology and Clinical Immunology (EAACI) and approved by the European Rhinologic Society (ERS), although that paper was published after the inclusion procedure for this study had started. Healthy volunteers were invited to participate in the study when visiting the Örebro travel consultation clinic between
2004 and 2006. All participants in both groups were legally of full age ( $>18$ years) and provided written informed consent to participate in the study. All procedures performed in this study were in accordance with the ethical standards of the national research committee and with the 1964 Helsinki declaration. The study was approved by the regional ethical review board in Uppsala, Sweden (ref: 2005:011/1).

\section{Sample collection and microbiological analyses}

Blood samples were collected from patients and controls, and serum was frozen pending further analysis. Information about growth of $S$. aureus in nares or maxillary sinus was available from a previous study ${ }^{(5)}$. Information about age, gender, acetylsalicylic acid (ASA) intolerance, asthma, nasal polyp, and smoking habits was collected. Sampling techniques and handling of specimens have been described previously ${ }^{(5)}$. In brief, bacterial samples were collected from the nares and maxillary sinuses of the patients, and from the nares of the healthy controls using a cotton-tipped aluminium swab (Copan, Brescia, Italy) passing the nasal cavity and placed into the sinus through the enlarged sinus ostium under visual control. This swab is of bendable aluminium and can be pre-curved, allowing it to be carefully inserted into the maxillary sinus. When collecting the maxillary sinus samples, care was taken to avoid contamination from contact with the nose wall. The culturing procedure and further species verification of bacterial growth were performed in accordance with routine diagnostic procedures at the Department of Laboratory Medicine, Clinical Microbiology, Örebro University Hospital. All controls and patients filled in a 6-parameter symptom score questionnaire from the Swedish Rhinologic Society, with answers given on a visual analog scale (VAS) comprising a stepless line symbolizing the range between "absence of symptoms" and "worst symptoms possible". This procedure was used in order to avoid clustering of scores around a preferred numeric value. A ruler was used to convert the respondents' markings to the corresponding numbers between 0 (no symptoms) and 10 (worst symptoms possible). Variables used were nasal congestion, frequency of nasal secretion, loss of smell, facial pain or pressure, coughing, and quality of life (in terms of fatigue). A total score was summed for each individual, with a maximal (worst) value of 60 points.

\section{Microarray based immunoglobulin analysis}

Serum samples were analysed using a protein array comprising 61 different $S$. aureus specific antigens covalently linked to the array surface. Information about the antigens can be extracted from Kloppot et al. ${ }^{(19)}$ and Selle at al. ${ }^{(20)}$. The S. aureus specific antigens were immobilized using different concentrations ranging from $0.01-0.5 \mathrm{mg} / \mathrm{ml}$. The protein arrays were read out using an ArrayMate and the data were analyzed using IconoClust software (both by Alere Technologies $\mathrm{GmbH}$, Germany). 
Table 1. Demographic data for 42 patients with chronic rhinosinusitis (CRS) with nasal polyposis (CRSwNP) and CRS without nasal polyposis (CRSsNP) and 57 healthy controls. Significant values $(a=0.05)$ are expressed in bold.

\begin{tabular}{|c|c|c|c|c|c|}
\hline Characteristics & $\begin{array}{l}\text { CRSwNP* } \\
n=24\end{array}$ & $\begin{array}{l}\text { CRSsNP** } \\
n=18\end{array}$ & $\begin{array}{l}\text { All CRS } \\
n=42\end{array}$ & $\begin{array}{c}\text { All controls*** } \\
n=57\end{array}$ & $\begin{array}{c}\text { P-value (all CRS vs. all } \\
\text { controls) }\end{array}$ \\
\hline Age, mean & $53(34-77)$ & $52(36-82)$ & $52.5(34-82)$ & $50(20-74)$ & 0.49 \\
\hline Male/female & $13 / 11$ & $4 / 14$ & $17 / 25$ & $26 / 31$ & 0.612 \\
\hline ASA intolerance, $n$ & $7(29 \%)$ & $4(22 \%)$ & $11(26 \%)$ & $3(5 \%)$ & 0.007 \\
\hline Asthma & $9(37 \%)$ & $3(17 \%)$ & $12(29 \%)$ & $5(9 \%)$ & 0.01 \\
\hline $\begin{array}{l}\text { Exposed to smoking } \\
\text { daily }\end{array}$ & $4(17 \%)$ & $6(33 \%)$ & $10(24 \%)$ & $6(10 \%)$ & 0.1 \\
\hline $\begin{array}{l}\text { No previous sinus } \\
\text { surgery }\end{array}$ & $21(87 \%)$ & 15 (83\%) & $36(86 \%)$ & $0(0 \%)$ & 0.00001 \\
\hline $\begin{array}{l}\text { Mean score on } 6 \text {-item } \\
\text { VAS (max } 60 \text { points) }\end{array}$ & 38.2 & $35.9^{* * * *}$ & 37.8 & 9.7 & $<0.00001$ \\
\hline
\end{tabular}

*one serum missing, ${ }^{* *}$ two sera missing, ${ }^{* * *}$ one serum missing, ${ }^{* * * *}$ two symptom scores missing

During read-out, relative signal intensities of defined regions (at predefined spot coordinates) on the Staph-Toxin-Array were determined. The normalized intensities (NI) of the spots were determined as $\mathrm{NI}=1-(\mathrm{M} / \mathrm{BG})$, where $\mathrm{M}$ is the average intensity of the spot and BG is the intensity of the local background. Hence, NI values ranged between 0 (no signal) and 1 (maximal intensity). Version 5.1 of the GraphPad Prism software package was used to visualize the protein array data as described elsewhere. It should be noted that the absorbance units for specific antigens could only be used to compare groups for the same antigen, and not for a comparison between different antigens, because different serum dilutions were applied for testing against different antigens.

\section{Statistical analysis}

The participants' demographic characteristics, symptom scores, and antibody measurements are presented using descriptive statistical methods. Continuous data are summarized as mean $\pm \mathrm{SD}$ or as median with corresponding interquartile range (IQR) where suitable, and categorical data are presented as percentages. Differences between groups were tested using Student's t-test or the Mann-Whitney U-test or the Kruskal-Wallis test for continuous data where suitable, and using a $\chi 2$ test or Fisher's exact test for categorical data. Spearman's correlation coefficient was used to evaluate the relationship between symptom scores and serum IgG levels. Holm-Bonferroni correction was applied to control for false discovery rate in multiple hypothesis tests ${ }^{(21)}$. A two-sided $p$-value $<0.05$ was considered statistically significant. All statistical analyses were conducted in version 22 of IBM SPSS Statistics. Figures for visualization of multivariate data were produced using MATLAB R2018a.

\section{Results}

Initially 43 patients with CRS and 58 healthy controls were enrolled. Of the controls one was excluded due to an age below 18 years and one patient was excluded due to having titanic dental implants reaching the maxillary sinus. For determining anti-staphylococcal lgG antibody responses to $S$. aureus antigens, sera were available from 39 of 42 CRS patients and 56 of 57 healthy controls; the remaining four sera were lost during processing. Demographic data are given in Table 1. Asthma and ASA intolerance were both significantly more frequent in the patient group. There was no information available about previous antibiotic treatment prior to inclusion, but none had antibiotics at the time of inclusion. Three patients had 7 days of oral corticosteroid treatment before surgery and inclusion in study. There were no patients with immunosuppressive disease in either group. Most of the patients had regular ongoing treatment with saline rinsing of the nose combined with nasal corticosteroids.

To study the $S$. aureus specific adaptive immune response, we focused on IgG antibodies directed toward staphylococcal components exposed to the immune system such as secreted proteins and antigens exposed on the surface of the bacteria, enterotoxins (TSST-1, SEB, SEC, SEl, SEK, SEL, SEM, SEQ), other secreted factors (Sbi, LukF-PV, HlgA, HlgB, HlgC, Hlb, SplA, SplB), and cell wall associated proteins (IsaA, Plc, Efb-C, SCIN). When comparing S. aureus specific serum IgG levels for all patients with CRS and all healthy controls, regardless of growth of $S$. aureus, we found statistically significant higher lgG antibody levels to LukF-PV (adjusted $\mathrm{p}=0.038$ ) and TSST-1 (adjusted $\mathrm{p}=0.02$ ) in CRS patients than in healthy controls. Antibody levels to SCIN differed between the two groups, but the difference was not statistically significant after Holm-Bonferroni correction (adjusted $p=0.792$ ) (Figure 1). No gender differences were found in antibody levels. Serum anti-TSST-1 levels were also statistically significantly higher for patients with S. aureus present in sinus compared to controls (adjusted $\mathrm{p}=0.02$ ), but antibodies to LukF-PV did not 
A

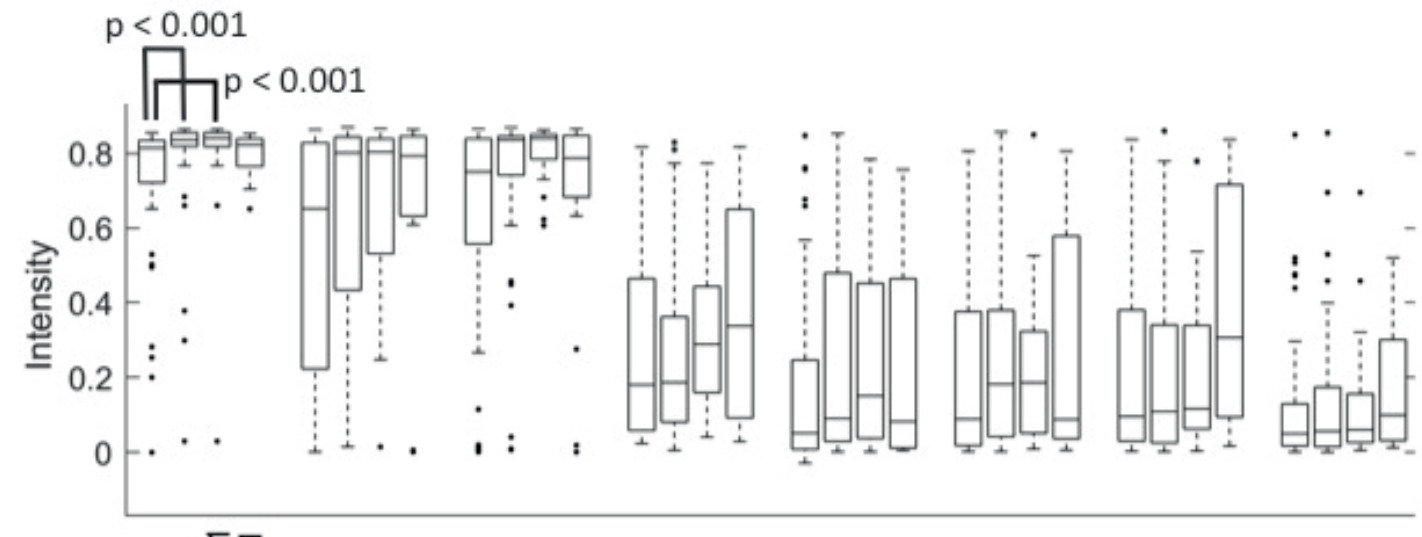

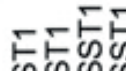

ए5ूक

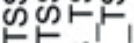

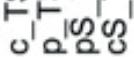

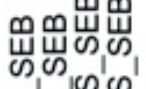

㟧出

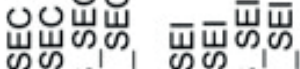

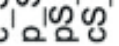

B

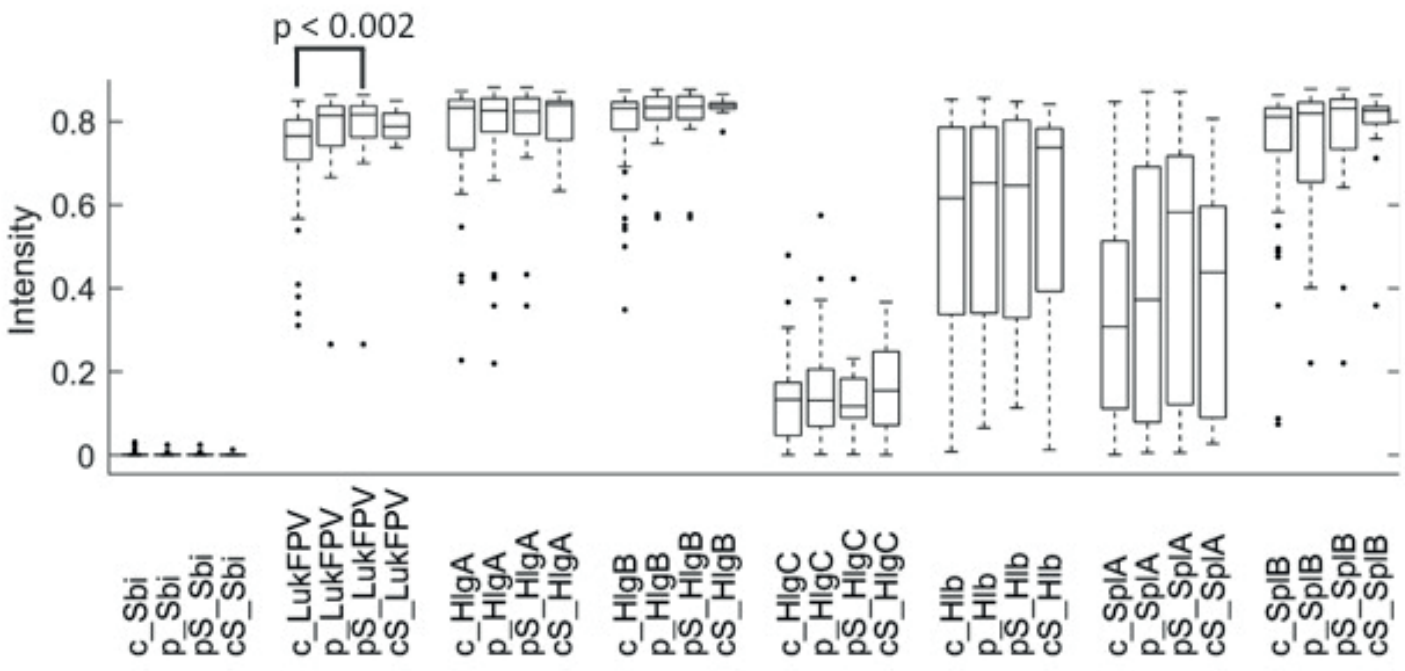

C

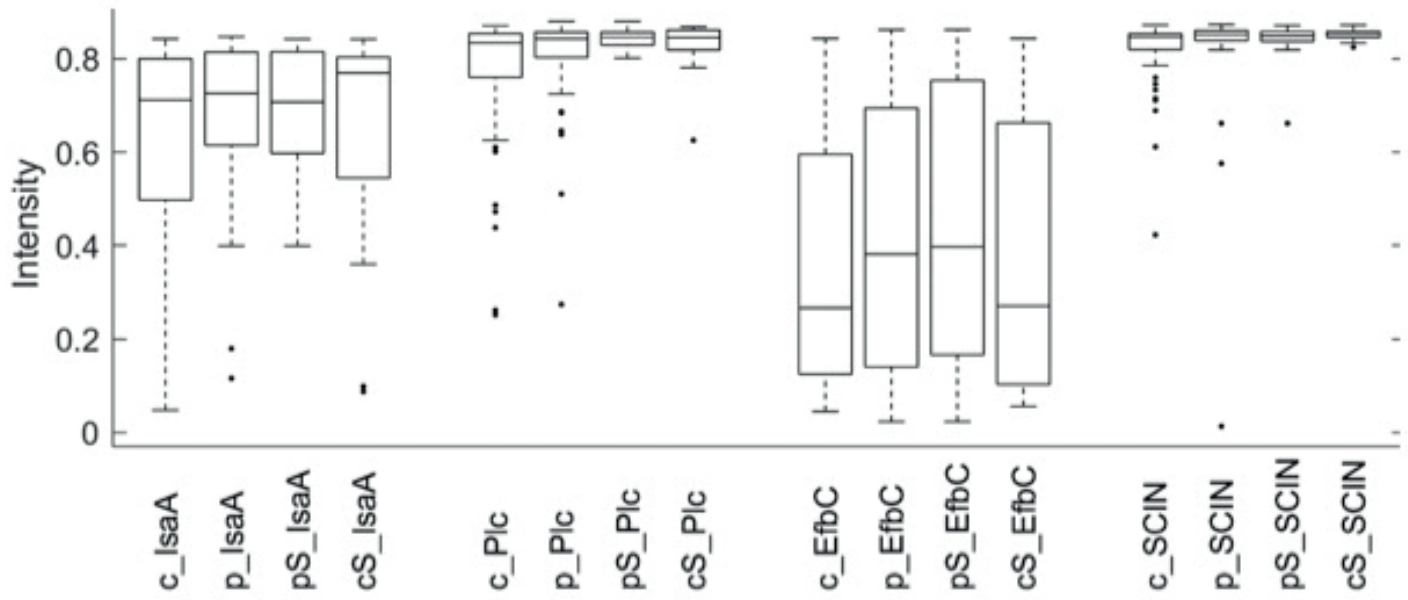

Figure 1. Anti-staphylococcal lgG relative intensity of selected S. aureus-specific antibodies in serum of 39 patients with CRS (p) and 56 healthy controls (c), including 22 CRS patients with growth of S. aureus in maxillary sinus (pS) and 17 controls with S. aureus in nares (cS), using the Staph-ToxinArray. TSST-1 and LukF-PV remained significant after Holm-Bonferroni correction as shown. 
A

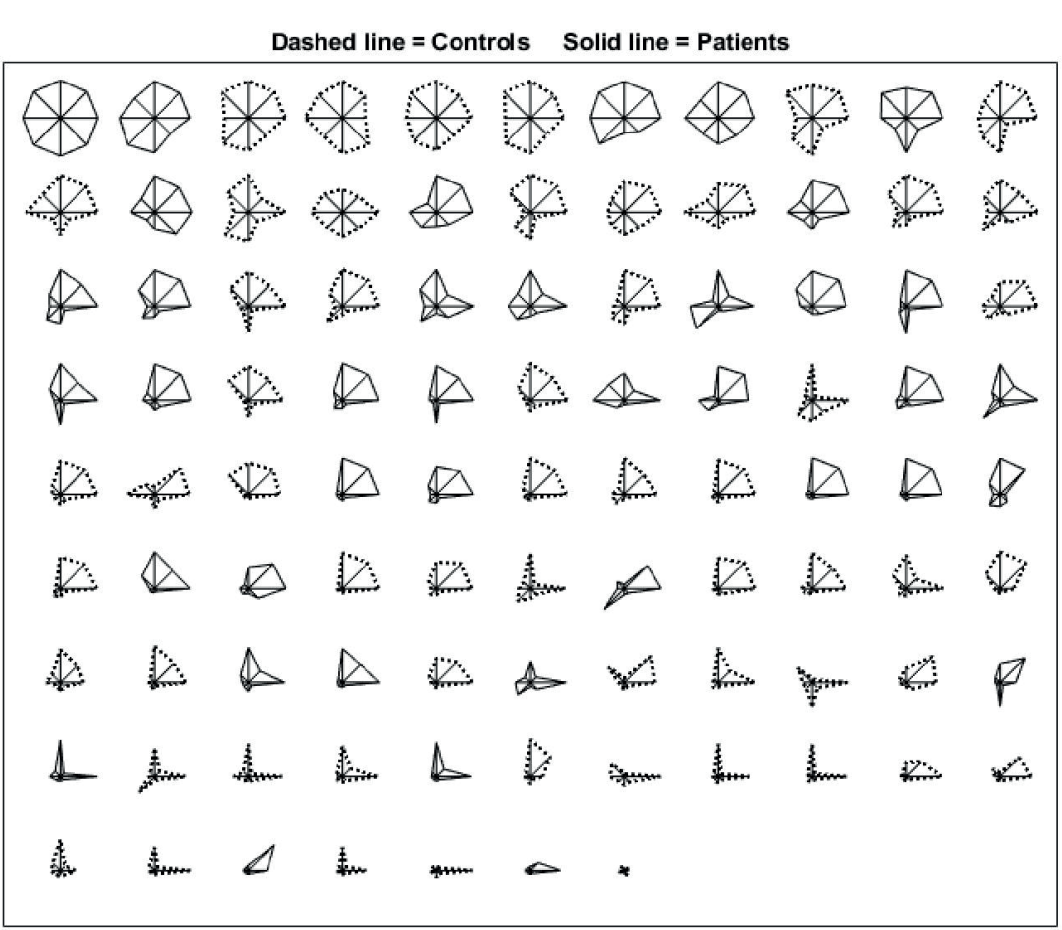

B

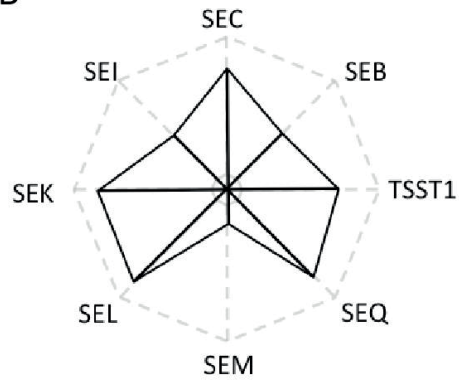

Figure 2. A) Multivariate visualization of anti-staphylococcal lgG levels in serum from 95 individuals (39 CRS patients and 56 controls) using glyph plots to show the signal intensities of staphylococcal enterotoxin antibodies (TSST-1, SEB, SEC, SEI, SEK, SEL, SEM, SEQ) in the Staph-Toxin-Array. B) An example showing the principle behind a glyph plot. Each glyph represents one individual, and each spoke shows the proportional antibody level of one enterotoxin. The outer dashed perimeter connecting the spokes shows the maximum antibody levels for the antigens found for all controls and patients and the inner perimeter shows the minimum values.

show a significant difference after correction with Holm-Bonferroni correction (adjusted $\mathrm{p}=0.057$ ) (Figure 1). When comparing anti-staphylococcal lgG antibody levels in serum from CRS patients with growth of $S$. aureus in both maxillary sinus and nares and from controls with growth of S. aureus in nares, there was no significant difference for any specific antigen. None of the S. aureus specific IgG levels in serum from CRS patients with growth of $S$. aureus in maxillary sinus differed significantly from the corresponding levels in CRS patients without growth of S. aureus in maxillary sinus. Furthermore, when comparing anti-staphylococcal lgG antibody levels in serum of controls with versus without growth of S. aureus in nares, there were no significance differences for any specific antigens, although some differences were very close to significance (SEM, SCIN, LukF-PV). When comparing anti-staphylococcal IgG antibodies for CRSwNP, CRSsNP and Controls there was statistically significant raised LukF-PV ( $p=0.04$, adjusted for multiple comparisons) but not for TSST-1 (adjusted $p=0.08$ ) or other antigens. In this comparison, CRSsNP showed the highest medians and controls the lowest. S. aureus specific IgG levels did not differ significantly between patients with CRSwNP and those with CRSsNP who displayed growth of S. aureus in their maxillary sinus. However, there was a significantly higher level of anti-staphylococcal IgG directed to LukF-PV (adjusted $\mathrm{p}=0.02$ ) in individuals (CRS and controls) with S. aureus in nares compared with those without growth of S. aureus in nares. The anti-staphylococcal lgG response for the 20 analysed specific antigens was generally higher in the patient group for each antigen, but the difference was not significant (Figure 1). When comparing anti-staphylococcal IgG response in CRSwNP, CRSsNP, and controls dependent on whether the individual had ASA intolerance, asthma, and exposure to smoking, respectively, no significant differences were found. A multivariate visualization of IgG levels in serum for all CRS patients and controls using a glyph plot showed highly variable levels of relative intensity for specific anti-staphylococcal lgG antibody in both healthy individuals and patients with CRS, and no groupspecific pattern could be seen (Figure 2). For 37 patients and 56 controls, both a serum sample and an individual self-reported VAS score were available. Scatter plots of serum antibody levels to TSST-1 and LukF-PV versus symptom score are shown in Figures 3 (patients and controls) and 4 (patients with and without growth of S. aureus in maxillary sinus). Spearman's correlation was used to assess the relationship between VAS score and antibody levels for TSST-1 or LukF-PV, and as expected, patients had higher symptom scores than controls $(p=0.00001)$. There was a weak correlation between antibody levels and symptom scores (Figures 3 and 4). 

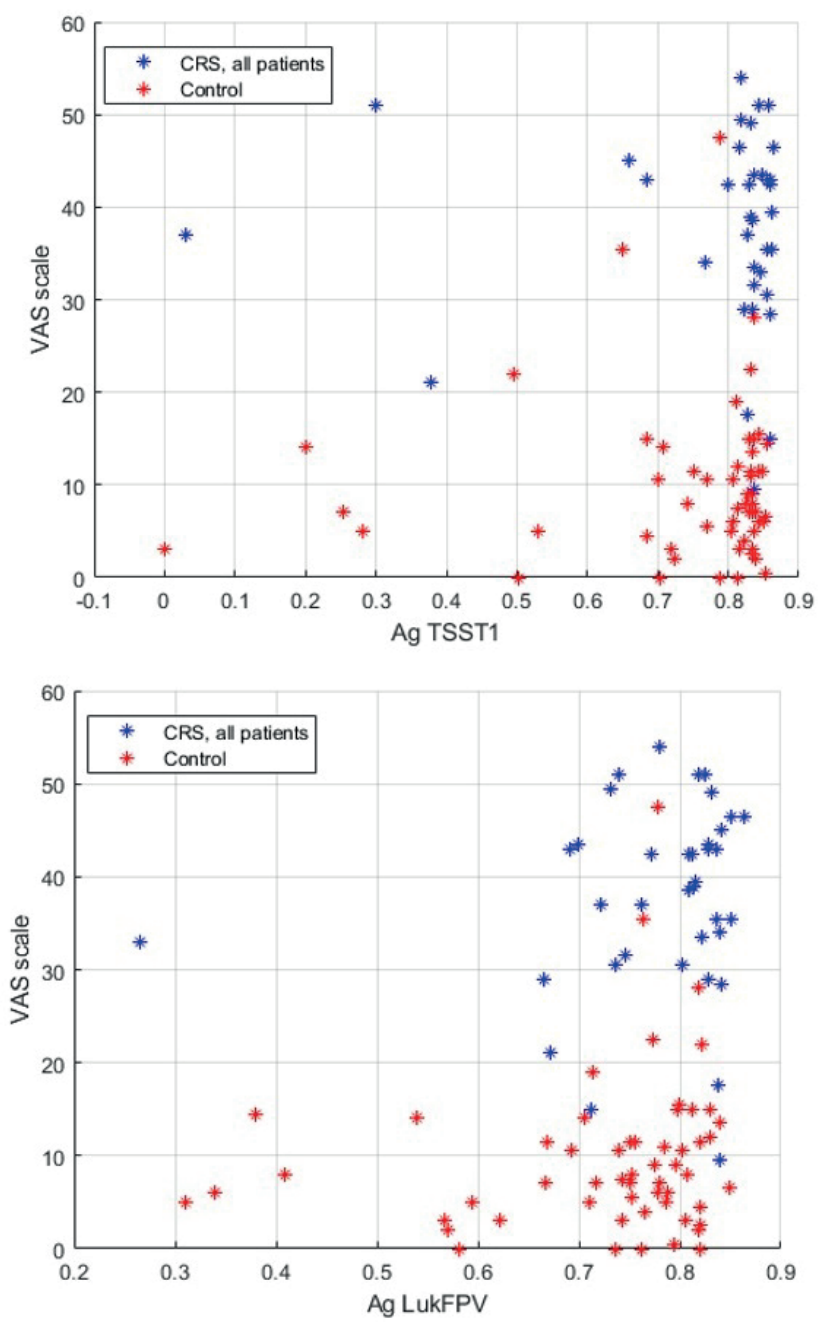

Figure 3. Scatter plot of serum antibody levels for TSST-1 and LukF-PV versus symptom score for CRS patients and healthy controls (Spearman's correlation coefficient rho). Anti-LukF-PV in CRS patients and symptom score shows rho $=0.113, p=0.507$. Anti-TSST- 1 in CRS patients and symptom score shows rho $=0.113, p=0.507$.

\section{Discussion}

CRS is a significant health problem ${ }^{(22)}$. The cause of the disease is not fully understood, and it is considered to be multifactorial; probably the result of a complex interaction between host, environment, and microbes. S. aureus has been suggested to have an influence on the pathogenesis ${ }^{(2)}$. A large meta-analysis, based on 12 case-control studies and a total of 340 cases and 178 controls, concluded that $S$. aureus superantigens may be a risk factor for the persistence and severity of CRSwNP (3). It is known that anti-staphylococcal antibody levels in serum contribute to protection, modification of the course, and reduced risk of complications of staphylococcal infections ${ }^{(23,24)}$, but little is known about their role in chronic inflammatory disease. A previous study showed highly elevated antibodies in nasal polyps in CRS compared to uncinate tissue from healthy
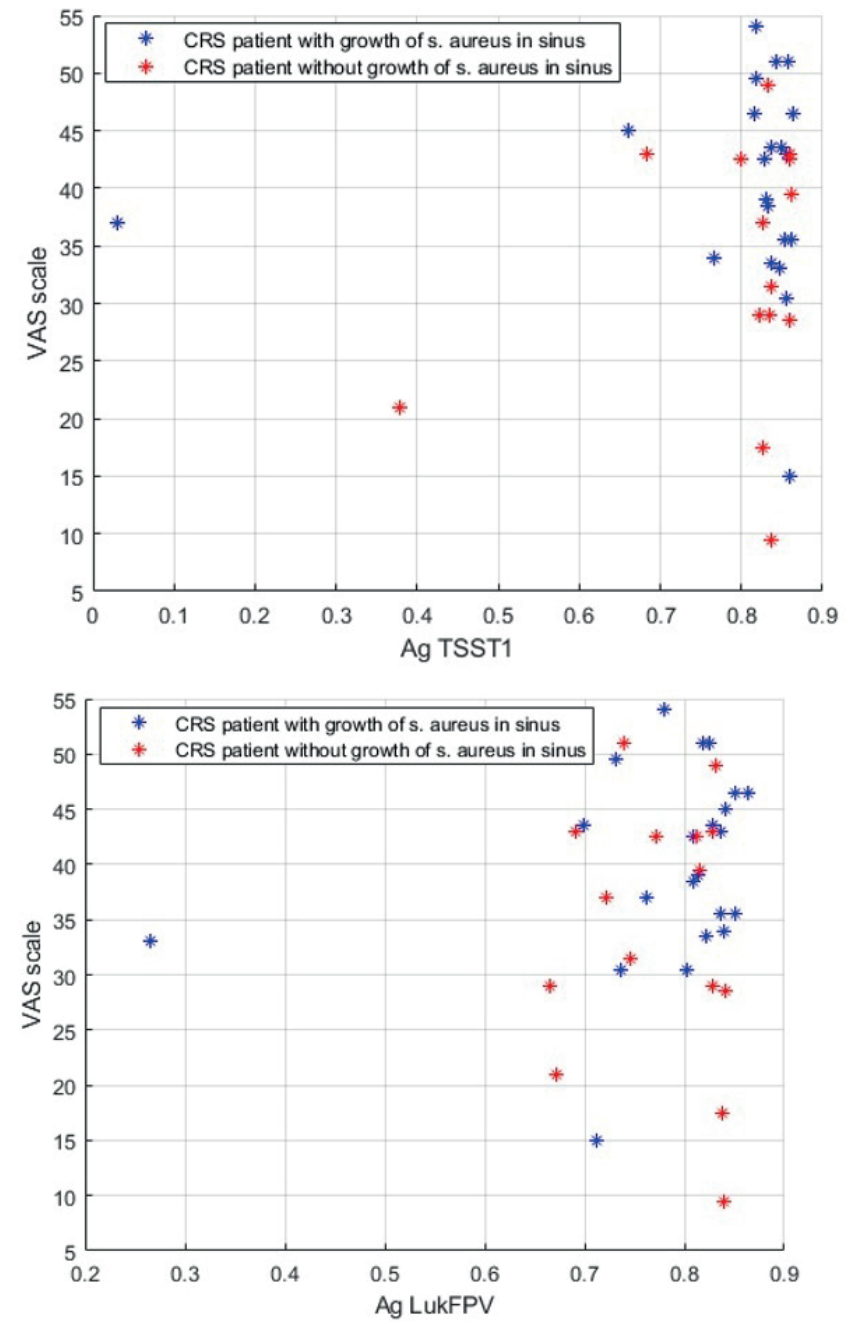

Figure 4. Scatter plot of serum antibody levels for TSST-1 and LukF-PV versus symptom score for CRS patients with and without $S$. aureus in sinus (Spearman's correlation coefficient rho). Anti-LukF-PV in CRS patients with $S$. aureus in sinus shows rho $=-0.026, p=0.91$. Anti-TSST- 1 in CRS with S. aureus in sinus shows rho $=-0.197, p=0.381$.

controls ${ }^{(25)}$. In another study by the same group, local autoantibodies in CRSwNP were elevated ${ }^{(26)}$. The understanding of the pathophysiology of CRS involve complex immunology. CRSwNP and CRSsNP seem to have different endotypes, with inflammation mediated as Th2 and Th1 reaction ${ }^{(27,28)}$. There is evidence for the involvement of S. aureus in CRSwNP, altering the cytokine response toward a Th2 reaction with eosinophilia, polyclonal local IgE production ${ }^{(2,29,30)}$. In the present study, we focused on IgG to investigate whether patients with CRS had different serum IgG antibody response to specific staphylococcal antigens than healthy controls, and especially toward staphylococcal enterotoxins. We also investigated the correlation between severity of symptoms and specific immune response. We found statistically significantly higher anti-staphylococcal IgG levels for LukF-PV and TSST-1 in CRS patients than in 
controls. In addition, when comparing specific anti-staphylococcal IgG antibody levels in CRS patients with growth of S. aureus in maxillary sinus versus healthy controls, we found statistically significant higher IgG levels toward TSST-1 in the CRS patients. In a previous study we showed a significantly higher presence of S. aureus in the nares of CRS patients than in controls, as well as a high presence of $S$. aureus in both maxillary sinus and nares of patients with CRS ${ }^{(5)}$. The immune system in CRS cases might be more exposed to TSST-1 and LukF-PV than the immune system in controls, resulting in a stronger lgG response. However, the presence of $S$. aureus in maxillary sinus did not have any impact on the anti-staphylococcal lgG levels in serum from CRS patients. There was no significant difference in antibody levels toward any of the tested staphylococcal antigens for CRS patients with and without growth of $S$. aureus in maxillary sinus or controls with and without S. aureus in nares. This finding could indicate a low importance of $S$. aureus in maxillary sinus for the disease. A probable stimulator for serum immunoglobulin B could be the presence of $S$. aureus in nares. We used Holm-Bonferroni correction to strengthen our comparisons of multiple variables. The lowest $\mathrm{p}$-values for differences in IgG levels in our comparisons, though not always significant after statistical correction, were found for TSST-1, LukF-PV, SCIN, PIc, and enterotoxins. It could be hypothesized that a larger study size might have resulted in significant differences. Panton-Valentine leukocidin (PVL) is a pore-forming cytotoxin composed of two components, LukF-PV and LukS-PV. These two components bind to neutrophilic cells causing lysis. PVL is associated with primary skin infection and necrotizing pneumonia ${ }^{(31)}$, but its role in chronic diseases such as CRS has not been evaluated. TSST-1 and staphylococcal enterotoxins act as superantigens. Van Belkum et al. showed that increased antibody levels exist in individuals persistently colonized with S. aureus ${ }^{(32)}$. Our study used one-time sampling, and so it was not possible to investigate the effect of persistent colonization. In general, we found higher levels of anti-staphylococcal lgG antibodies in serum from patients with CRS compared to healthy controls, and also in CRS patients with growth of $S$. aureus in maxillary sinus compared to healthy controls, which could reflect more persistent colonization in patients with CRS or a presence of $S$. aureus strains more prone to produce antigens that promote antibody response. However, previous staphylococcal infection could affect the serum level of antibodies. We found highly variable levels of specific anti-staphylococcal lgG antibody levels towards staphylococcal antigens in both healthy individuals and patients with CRS, and as can be seen in the glyph plot (Figure 2), no contexture of immunoglobulin composition was repetitive for these groups. One limitation is that we do not know how specific anti-staphylococcal antibodies varies over time in a normal population. A retrospective study investigating the role of specific antibody deficiency in 129 CRS patients with medically refractory CRS ${ }^{(33)}$ found low baseline anti-pneumococcal antibody levels in $72 \%$ of the patients, and of these $11.6 \%$ of these showed an inadequate response to vaccination with 23-valent unconjugated pneumococcal vaccine. Studies regarding anti-staphylococcal lgG antibody levels toward specific staphylococcal antigens in CRS are limited. A recent meta-analysis including 1418 individuals with CRS from 13 studies reported that $23 \%$ of patients with difficult-to-treat CRS and $13 \%$ of individuals with recurrent CRS had immunoglobulin deficiencies ${ }^{(15)}$. We found generally higher antibody levels toward S. aureus antigens in CRS cases than controls. S. aureus specific lgG levels did not differ significantly between patients with CRSwNP and CRSsNP. A larger study size would have made it possible to investigate any differences in IgG levels between patients with CRSwNP and those with CRSsNP. What effect LukF-PV and TSST-1 have in the paranasal mucosa is not clear. IgE response to superantigens has been shown locally in nasal polyps. IgE production results in an allergic response to staphylococcal antigen, eosinophilic leukocyte reaction and Th2 cytokine mediated reaction. The presence of $\mathrm{lgG}$ is interpreted as reflecting exposure to a specific antigen and a readiness of the immune system to protect. Much research has been done in the field of CRS, S. aureus and superantigens by Bernstein et al. and Bachert et al. suggesting an impact of S. aureus in CRS ${ }^{(4,34,}$ ${ }^{35)}$. The raised levels of TSST- 1 and LukF-PV antibodies might indicate a relevance of these enterotoxins in the pathogenesis of CRS disease. It is interesting that it is the staphylococcal antigens TSST-1 and PVL, according to the significant antibodies demonstrated in the present study, that the CRS patients actually have been exposed to and display a immune response against. especially since these exotoxins display superantigenic properties and may induce and maintain chronic inflammation. Holm-Bonferroni correction for multiple comparison was applied to all data to control for false discovery rate in multiple hypothesis tests, and this strengthens our findings. We used a 6-parameter symptom score for CRS patients and controls to evaluate severity of symptoms correlated to anti-staphylococcal IgG levels. A similar symptom score has been used in a previous study ${ }^{(36)}$. Not surprisingly, the patients had a significantly higher symptom score than controls. The scoring of symptoms was considered as a measure of severity of disease. There were no correlations between symptom score and IgG levels for LukF-PV or TSST-1 when comparing values from all patients and controls, and the presence of $S$. aureus in sinus had no impact on severity of symptoms. Additional clinical data such as information on antibiotic therapy for the patients and/or administration of corticosteroids or antibiotic therapy for the control group would have been of value, as these factors might have affected the results. The presence of staphylococcal antigens in or at the mucous membranes of the sinonasal tract could be an valuable aspect for future investigation, as could analyses of local 
cytokine and IgG antibody response to TSST-1 and LukF-PV in the mucosa.

\section{Conclusions}

Anti-staphylococcal lgG antibody levels to TSST-1 and LukF-PV were significantly higher in CRS patient group than in healthy controls. The levels of anti-TSST- 1 antibodies were also significantly higher in the CRS patients group with S. aureus in the maxillary sinus than in controls. There were no correlations between the severity of symptoms and serum anti-staphylococcal IgG antibody levels for LukF-PV and TSST-1, respectively, that could be explained by existing CRS disease. There was no correlation between severity of symptoms and $S$. aureus growth in the maxillary sinus for the CRS group. A larger study size would be desirable. Anti-TSST-1 and anti-LukF-PV could be interesting markers for future studies of CRS and the raised levels of antibodies specific to TSST-1 and LuKF-PV might indicate a relevance of these enterotoxins in the pathogenesis of CRS disease.

\section{Acknowledgement}

This study was supported by research Committee of Region Örebro County, Sweden and ALF funding for Region Örebro län, Sweden.

\section{Authorship contribution}

BS, UT, HF and SH participated in the conception and design of the study. UT and $\mathrm{SH}$ recruited the patients and healthy volunteers. UT and SH carried out the data acquisition. RE, SE and SM designed and analyzed the experiments. EM carried out the experiments. YC and UT were involved in the computational statistical analysis of the data. BS, UT and SH interpreted the results. UT drafted the manuscript. All authors read and revised the manuscript and also approved the final version.

\section{Conflict of interest}

Elke Müller, Stefan Monecke, and Ralf Ehricht are employees of Abbott (Alere Technologies $\mathrm{GmbH}$ ), the company that manufactures the microarrays used for this study. This does not affect the authors' adherence to journal policies on sharing data and materials. Abbott (Alere Technologies $\mathrm{GmbH}$ ) provided support in the form of salaries for these authors, but did not have any additional role in the study design, data collection and analysis, decision to publish, or preparation of the manuscript. All other authors declare no competing interest.

\section{Ethics approval and consent to participate}

All procedures performed in this study were in accordance with the ethical standards of the national research committee and with the 1964 Helsinki declaration. The study was approved by the regional ethical review board in Uppsala, Sweden (ref: 2005:011/1).

\section{Consent for publication}

Not applicable

\section{Availability of data and materials}

The datasets used and analysed during the current study are available from the corresponding author on reasonable request.

\section{Funding}

This study was funded by the Research Committee of Örebro County Council, Sweden, and ALF funding for Region Örebro County, Sweden. The funding was used to finance time for research work and analysis.

\section{References}

1. Hastan D, Fokkens WJ, Bachert C et al Chronic rhinosinusitis in Europe--an underestimated disease. GA(2)LEN study. Allergy 2011; 66(9):1216-23.

2. Bachert C, Holtappels G. Pathophysiology of chronic rhinosinusitis, pharmaceutical therapy options. GMS Curr Top Otorhinolaryngol Head Neck Surg 2015; 14: Doc09.

3. Ou J, Wang J, Xu Y, Tao ZZ, Kong YG, Chen SM, et al. Staphylococcus aureus superantigens are associated with chronic rhinosinusitis with nasal polyps: a metaanalysis. Eur Arch Otorhinolaryngol 2014; 271(10):2729-36.

4. Bernstein JM, Kansal R. Superantigen hypothesis for the early development of chronic hyperplastic sinusitis with massive nasal polyposis. Curr Opin Otolaryngol Head Neck Surg 2005; 13(1):39-44.

5. Thunberg U, Soderquist B, Hugosson $S$.
Bacterial findings in optimised sampling and characterisation of S. aureus in chronic rhinosinusitis. Eur Arch Otorhinolaryngol 2017; 274(1):311-9.

6. Biel MA, Brown CA, Levinson RM et al Evaluation of the microbiology of chronic maxillary sinusitis. Ann Otol Rhinol Laryngol 1998; 107(11 Pt 1):942-5.

7. Wertheim HF, Melles DC, Vos MC et al. The role of nasal carriage in Staphylococcus aureus infections. Lancet Infect Dis 2005; 5(12):751-62.

8. Kluytmans J, van Belkum A, Verbrugh $\mathrm{H}$. Nasal carriage of Staphylococcus aureus: epidemiology, underlying mechanisms, and associated risks. Clin Microbiol Rev 1997 10:505-20.

9. Breuer K, Kapp A, Werfel T. Bacterial infections and atopic dermatitis. Allergy 2001; 56 (11):1034-1041.

10. Jappe U. Superantigens and their association with dermatological inflammatory dis- eases: facts and hypotheses. Acta Derm Venereol 2000; 80(5):321-8.

11. Tomassen $P$, Jarvis $D$, Newson $R$, Van Ree $R$ Forsberg B, Howarth P, et al. Staphylococcus aureus enterotoxin-specific $\lg$ E is associated with asthma in the general population: a GA(2)LEN study. Allergy 2013; 68(10):128997.

12. Yang PC, Liu T, Wang BQ et al. Rhinosinusitis derived Staphylococcal enterotoxin B possibly associates with pathogenesis of ulcerative colitis. BMC Gastroenterol 2005; 5:28.

13. Bachert C, van Zele T, Gevaert P, De Schrijver $L$, Van Cauwenberge P. Superantigens and nasal polyps. Curr Allergy Asthma Rep 2003; 3(6):523-31.

14. Alqudah M, Graham SM, Ballas ZK. High prevalence of humoral immunodeficiency patients with refractory chronic rhinosinusitis. Am J Rhinol Allergy 2010; 24(6):409-12.

15. Schwitzguebel AJ, Jandus P, Lacroix JS, Seebach JD, Harr T. Immunoglobulin defi- 
ciency in patients with chronic rhinosinusitis: systematic review of the literature and meta-analysis. J Allergy Clin Immunol 2015; 136(6):1523-31.

16. Odat H, Alqudah M. Prevalence and pattern of humoral immunodeficiency in chronic refractory sinusitis. Eur Arch Otorhinolaryngol 2016; 273(10):3189-93

17. van Belkum A, Melles DC, Nouwen J et al. Co-evolutionary aspects of human colonisation and infection by Staphylococcus aureus. Infect Genet Evol 2009; 9(1):32-47.

18. Fokkens W, Lund V, Bachert C et al. EAACl position paper on rhinosinusitis and nasal polyps executive summary. Allergy 2005: 60(5): 583-601.

19. Kloppot P, Selle M, Kohler $C$ et al. Microarray-based identification of human antibodies against Staphylococcus aureus antigens. Proteomics Clin Appl 2015; 9(11 12):1003-11.

20. Selle $M$, Hertlein $T$, Oesterreich $B$ et al. Global antibody response to Staphylococcus aureus live-cell vaccination. Sci Rep 2016; 6: 24754.

21. Holm S. A simple sequentially rejective multiple test procedure. Scand J Stat 1979; 6 (2):65-70.

22. Durr DG, Desrosiers MY, Dassa C. Impact of rhinosinusitis in health care delivery: the Quebec experience. J Otolaryngol 2001; 30(2):93-7.

23. Soderquist B, Danielsson D, Holmberg $H$ Vikerfors T. Granulocyte colony-stimulating factor (G-CSF) and interleukin (IL)-8 in sera from patients with Staphylococcus aureus septicemia. Clin Microbiol Infect 1995: 1(2):101-109.

24. Verkaik NJ, de Vogel CP, Boelens HA el al. Anti-staphylococcal humoral immune response in persistent nasal carriers and noncarriers of Staphylococcus aureus. Infect Dis 2009; 199:625-32.

25. Hulse KE, Norton JE, Suh L et al. Chronic rhinosinusitis with nasal polyps is characterized by B-cell inflammation and EBVinduced protein 2 expression. J Allergy Clin Immunology 2013; 131 (4) :1075-1083,1083 e1071-1077.

26. Tan BK, Peters AT, Schleimer RP, Hulse KE. Pathogenic and protective roles of $B$ cells and antibodies in patients with chronic rhinosinusitis. J Allergy Clin Immunol 2018; 141(5):1553-60.

27. Bachert C, Akdis CA (2016) Phenotypes and emerging endotypes of chronic rhinosinusitis. J Allergy Clin Immunol Pract 2016; 4 (4):621-628.

28. Tomassen $\mathrm{P}$, Vandeplas $\mathrm{G}$, Van Zele $T$ et al. Inflammatory endotypes of chronic rhinosinusitis based on cluster analysis of biomarkers. J Allergy Clin Immunol 2016; 137 (5):1449-1456 e1444.

29. Bernstein JM, Allen C, Rich G et al. Further observations on the role of Staphylococcus aureus exotoxins and IgE in the pathogenesis of nasal polyposis. Laryngoscope 2011: 121(3):647-55.

30. Bachert C, Gevaert P, Holtappels G, Johansson SG, van Cauwenberge P. Total and specific lgE in nasal polyps is related to local eosinophilic inflammation. J Allergy Clin Immunol 2001; 107(4):607-14.

31. Lina $G$, Piemont $Y$, Godail-Gamot $F$ et al Involvement of Panton-Valentine leukocidin-producing Staphylococcus aureus in primary skin infections and pneumonia. Clin Infect Dis 1999; 29(5):1128-1132

32. van Belkum A, Verkaik NJ, de Vogel CP et al. Reclassification of Staphylococcus aureus nasal carriage types. J Infect Dis 2009; 199(12):1820-6.

33. Carr TF, Koterba AP, Chandra R et al. Characterization of specific antibody deficiency in adults with medically refractory chronic rhinosinusitis. Am J Rhinol Allergy 2011; 25 (4):241-244.

34. Zhang N, Gevaert P, van Zele T et al. An update on the impact of Staphylococcus aureus enterotoxins in chronic sinusitis with nasal polyposis. Rhinology 2005; 43(3): $162-$ 168.

35. Derycke L, Perez-Novo C, Van Crombruggen K, Corriveau MN, Bachert C. Staphylococcus aureus and chronic airway disease. World Allergy Organ J 2010; 3 (8):223-228.

36. Abebola SO, Abidoye B, Ologe FE, Abebola OE, Oyejola BA. Health-related quality of life and its contributory factors in allergic rhinitis patients in Nigeria. Auris Nasus Larynx 2016: 43:171-175.

Ulrica Thunberg

Department of Otorhinolaryngology,

Örebro University Hospital

SE 70185 Örebro

Sweden

Tel: +46-196021441

Fax: +46-196023290

E-mail:

ulrica.thunberg@regionorebrolan.se 